\title{
Virtual Iraq: Initial Case Reports from a VR Exposure Therapy Application for Combat-Related Post Traumatic Stress Disorder
}

\author{
Albert A. Rizzo*, Ken Graap, Robert N. Mclay, Karen Perlman, Barbara O. Rothbaum, Greg Reger, \\ Thomas Parsons, JoAnn Difede and Jarrell Pair* Member, IEEE*
}

\begin{abstract}
Post Traumatic Stress Disorder (PTSD) is reported to be caused by traumatic events that are outside the range of usual human experience including (but not limited to) military combat, violent personal assault, being kidnapped or taken hostage and terrorist attacks. Initial data suggests that at least 1 out of 6 Iraq War veterans are exhibiting symptoms of depression, anxiety and PTSD. Virtual Reality (VR) delivered exposure therapy for PTSD has been used with reports of positive outcomes. The aim of the current paper is to present the rationale and brief description of a Virtual Iraq PTSD VR therapy application and present initial findings from two successfully treated patients. The VR treatment environment was created via the recycling of virtual graphic assets that were initially built for the U.S. Army-funded combat tactical simulation scenario and commercially successful $X$-Box game, Full Spectrum Warrior, in addition to other available and newly created assets. Thus far, Virtual Iraq consists of a series of customizable virtual scenarios designed to represent relevant Middle Eastern VR contexts for exposure therapy, including a city and desert road convoy environment. User-centered design feedback needed to iteratively evolve the system was gathered from returning Iraq War veterans in the USA and from a system deployed in Iraq and tested by an Army Combat Stress Control Team. Clinical trials are currently underway at Camp Pendleton and at the San Diego Naval Medical Center and the results from two successfully treated patients are presented along with a delineation of our future plans for research and clinical care using this application.
\end{abstract}

\section{INTRODUCTION}

$\mathrm{W}$ ar is one of the most challenging situations that a human being can experience. The physical, emotional, cognitive and psychological demands of a combat environment place enormous stress on even the bestprepared military personnel. The first systematic study of

Manuscript received July 15, 2007. This work was supported in part by the Office of Naval Research under Grant S-2005-ONR-02.

A.A. Rizzo, T. Parsons and J. Pair are with the University of Southern California Institute for Creative Technologies, Marina del Rey, CA 13274 USA, (corresponding author phone: 213-610-4737; fax: 310-574-5725; emails: ARizzo@usc.edu and Pair@ict.usc.edu,TParsons@ict.usc.edu).

K. Graap is with Virtually Better Inc., 2450 Lawrenceville Highway, Decatur, GA. 30033 USA (e-mail: Graap@virtuallybetter.com)

R.N. McLay and K. Perlman are with the Naval Medical Center - San Diego, 34800 Bob Wilson Drive, San Diego, CA 92134 USA (e-mails: Robert.Mclay@med.navy.mil and Karen.Perlman@med.navy.mil).

B.O. Rothbaum is with Emory University School of Medicine, 1841 Clifton Road, NE, Atlanta, GA 30329, USA (e-mail: BRothba@emory.edu).

J. Difede is with Weill Cornell Medical College, 525 East 68th Street, Box 200, New York, New York 10021 USA (email: jdifede@med.cornell.edu)

G. Reger is at the Madigan Army Medical Center, Fort Lewis, WA. USA (e-mail: greg.reger@us.army.mil) mental health problems due to the Iraq/Afghanistan conflicts revealed that "...The percentage of study subjects whose responses met the screening criteria for major depression, generalized anxiety, or PTSD was significantly higher after duty in Iraq (15.6 to 17.1 percent) than after duty in Afghanistan (11.2 percent) or before deployment to Iraq (9.3 percent)" [1]. These estimates were made before the violence escalated even further in the last 2 years and other reports since the original Hoge et al., (2004) publication, have indicated equivalent or higher numbers of returning military personnel reporting positive for PTSD and symptoms of other forms of mental disorders [2-3].

According to the DSM-IV [4], PTSD is caused by traumatic events that are outside the range of usual human experiences such as military combat, violent personal assault, being kidnapped or taken hostage, rape, terrorist attack, torture, incarceration as a prisoner of war, natural or man-made disasters, automobile accidents, or being diagnosed with a life-threatening illness. The disorder also appears to be more severe and longer lasting when the event is caused by human means and design (bombings, shootings, combat, etc.). Such incidents would be distressing to almost anyone, and is usually experienced with intense fear, terror, and helplessness. Typically, the initiating event involves actual or threatened death or serious injury, or other threat to one's physical integrity; or the witnessing or awareness of an event that involves death, injury, or a threat to the physical integrity of another person. The essential feature of PTSD is the development of characteristic symptoms that may include: intrusive thoughts and flashbacks, avoidance of reminders of the traumatic event, emotional numbing, hyperalertness, anger, isolation, anxiety, depression, substance abuse, survivor guilt, suicidal feelings and thoughts, negative self-image, memory impairment, problems with intimate relationships, emotional distance from family and others and denial of social problems. Symptoms of PTSD are often intensified when the person is exposed to stimulus cues that resemble or symbolize the original trauma in a non-therapeutic setting. Such uncontrolled cue exposure may lead the person to react with a survival mentality and mode of response that could put them and others at considerable risk.

Among the many approaches that have been used to treat PTSD, graduated exposure therapy appears to have the bestdocumented therapeutic efficacy [5,6]. Such treatment typically involves the graded and repeated imaginal reliving and narrative recounting of the traumatic event within the therapeutic setting. This approach is believed to provide a 
low-threat context where the patient can begin to therapeutically process the emotions that are relevant to the traumatic event as well as reconditioning the learning cycle of the disorder via a habituation/extinction process. While the efficacy of imaginal exposure has been established in multiple studies with diverse trauma populations [5-8], many patients are unwilling or unable to effectively visualize the traumatic event. In fact, avoidance of reminders of the trauma is one of the cardinal symptoms of PTSD. It is often reported that, "....some patients refuse to engage in the treatment, and others, though they express willingness, are unable to engage their emotions or senses." [9]. Research on this aspect of PTSD treatment suggests that the inability to emotionally engage (in imagination) is a predictor for negative treatment outcomes [10]. To address this problem, researchers have recently turned to the use of Virtual Reality (VR) to deliver exposure therapy by immersing clients in simulations of trauma-relevant environments that can more effectively deliver the controlled exposure to trauma cues needed for therapeutic gain. Such use and value of VR for the treatment of cognitive, emotional, psychological and motor disorders has been well specified $[11,12]$. This idea has been supported by three studies in which patients with PTSD were unresponsive to previous imaginal exposure treatments, but went on to respond successfully to VR exposure therapy $[7,9,13]$. The enthusiasm that is common among proponents of VR for this treatment is based on the view that VR has the capacity to deliver specific, consistent and controllable stimulus environments that don't rely on the hidden world of the patient's imagination.

In 1997, researchers at Georgia Tech released the first version of the Virtual Vietnam VR scenario for use as a graduated exposure therapy treatment for Post Traumatic Stress Disorder (PTSD) with Vietnam veterans [7]. This occurred over 20 years following the end of the Vietnam War. During those intervening years, in spite of valiant efforts to develop and apply traditional psychotherapeutic approaches to PTSD, the progression of the disorder in some veterans significantly impacted their psychological well being, functional abilities and quality of life, as well as that of their family members and friends. The tragic nature of this disorder also had significant ramifications for the U.S. Dept. of Veteran Affairs healthcare system often leading to designations of lifelong service connected disability status. The Virtual Vietnam scenario landmarked the first time that VR was applied to the treatment of PTSD and this initial effort produced encouraging results.

The first use of Virtual Vietnam with a veteran with PTSD was reported in a case study of a 50-year-old, Caucasian male veteran meeting DSM-IV criteria for PTSD [14]. Results indicated post-treatment improvement on all measures of PTSD and maintenance of these gains at a 6month follow-up. This case study was followed by an open clinical trial of VR for Vietnam veterans [7]. In this study, 16 male PTSD patients were exposed to two head mounted display (HMD)-delivered virtual environments, a virtual clearing surrounded by jungle scenery and a virtual Huey helicopter, in which the therapist controlled various visual and auditory effects (e.g. rockets, explosions, day/night, yelling). After an average of 13 exposure therapy sessions over 5-7 weeks, there was a significant reduction in PTSD and related symptoms. Similar positive results have also recently been reported for VR applied to PTSD resulting from the attack on the World Trade Center [9] in a case study using VR to provide re-exposure to the trauma with a patient who had failed to improve with traditional exposure therapy. These researchers have now reported positive treatment outcomes from a wait-list controlled VR study with patients who were not successful in previous imaginal therapy [13]. Such early results suggest that VR may be a valuable technology to apply for the treatment of PTSD and that it may be a promising component of a comprehensive treatment approach for persons with combat-related PTSD.

With this history in mind, the USC Institute for Creative Technologies (ICT) has created an immersive virtual reality system for exposure therapy with combat-related PTSD. The treatment environment is based on a creative approach to recycling virtual assets that were initially built for the commercially successful X-Box game and tactical training simulation scenario, Full Spectrum Warrior. As well, other existing and newly created assets available to ICT have been integrated into this rapidly evolving application. The presence of ICT expertise in the design of combat simulations and an interdisciplinary collaboration with leading experts in the field of PTSD has led to the opportunity to apply VR for this clinical challenge, albeit within a tighter timeframe than the technology allowed for Vietnam era veterans with PTSD.

The Virtual Iraq application consists of a series of virtual scenarios designed to represent relevant contexts for VR exposure therapy, including middle-eastern themed city and desert road environments (See Figures 1-6). In addition to the visual stimuli presented in the VR HMD, directional 3D audio, vibrotactile and olfactory stimuli of relevance can be delivered. The presentation of additive, combat-relevant stimuli in the VR scenarios can be controlled by a clinician via a separate "wizard of oz" interface, while the clinician is in full audio contact with the patient (See Figure 7). The clinical interface is a key feature in that it provides a clinician with the capacity to customize the therapy experience to the individual needs of the patient. The clinician can place the patient in VR scenario locations that resemble the setting in which the traumatic events initially occurred and can gradually introduce and control real time "trigger" stimuli (visual, auditory, olfactory and tactile) as is required to foster the anxiety modulation needed for therapeutic habituation. A full description of the application can be found in [15]. 


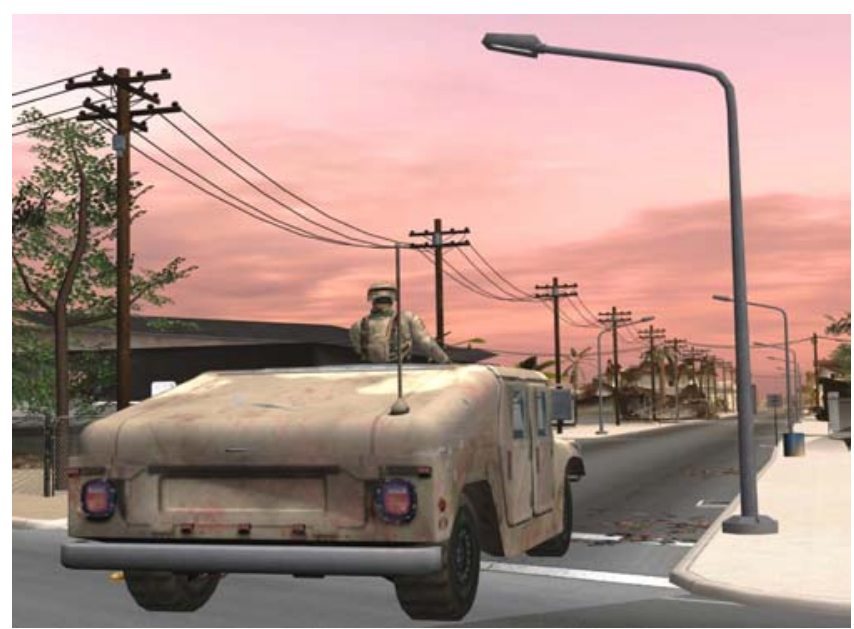

Fig. 1. Outskirts of Virtual Iraq City

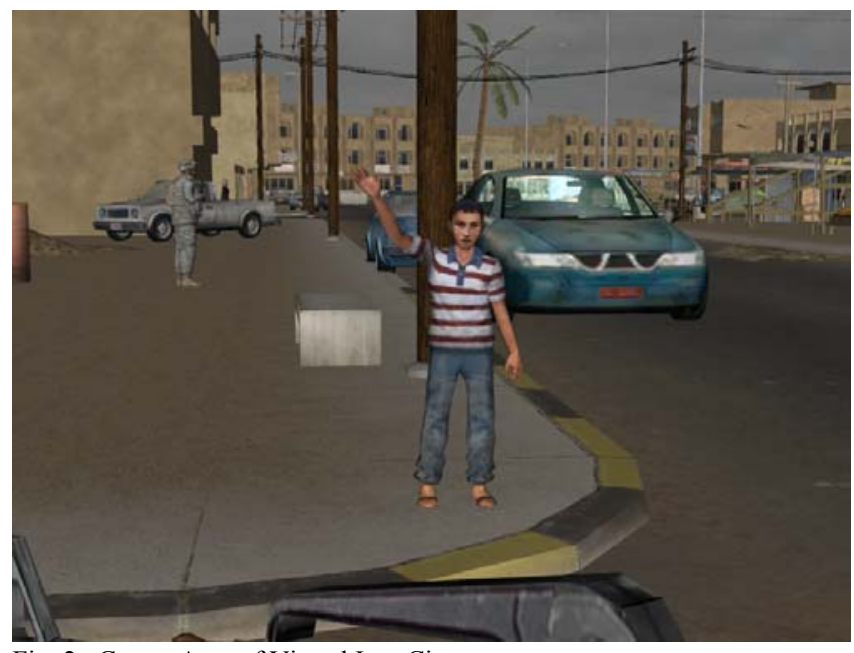

Fig. 2. Center Area of Virtual Iraq City

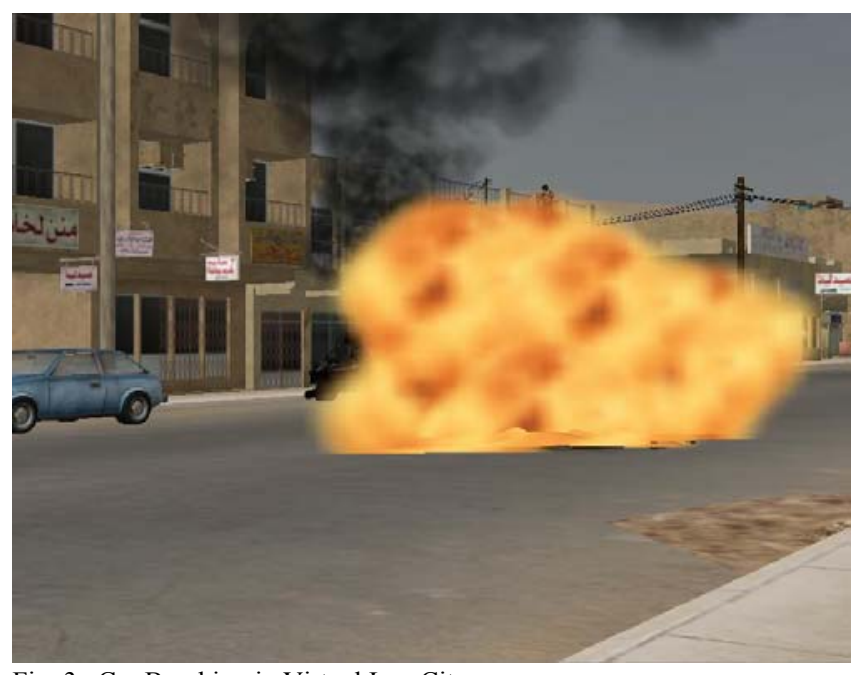

Fig. 3. Car Bombing in Virtual Iraq City

User-Centered tests with the application were conducted at the Naval Medical Center-San Diego and within an Army Combat Stress Control Team in Iraq (See Figure 8). This feedback provided by non-diagnosed Iraq-experienced military personnel provided information on the content and

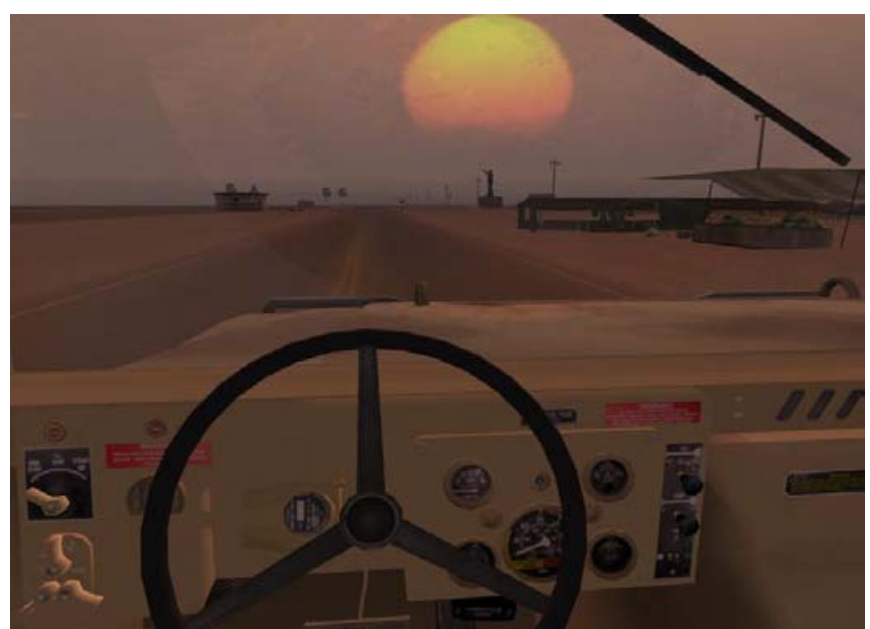

Fig. 4. Interior view from of Desert Road Humvee Scenario

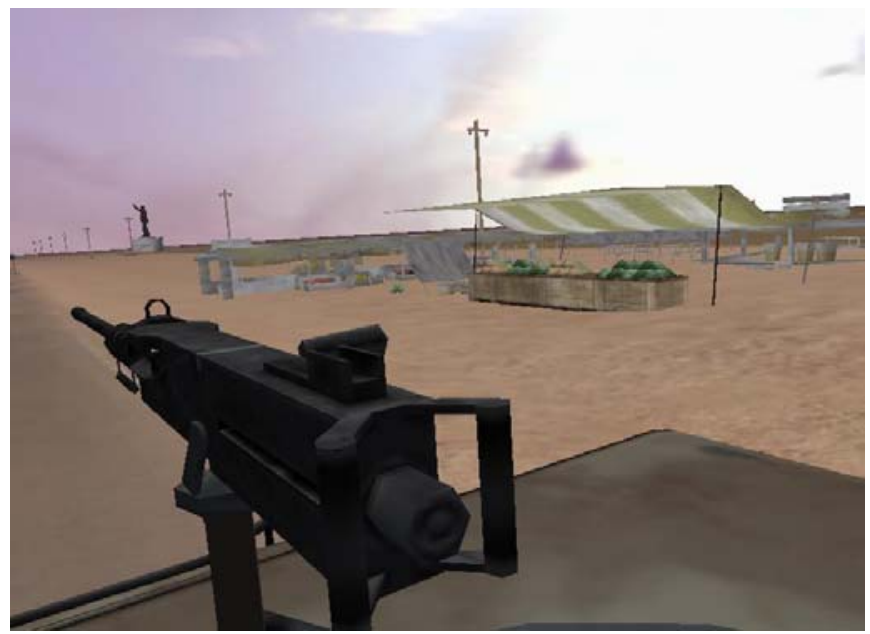

Fig. 5. Turret view from of Desert Road Humvee Scenario

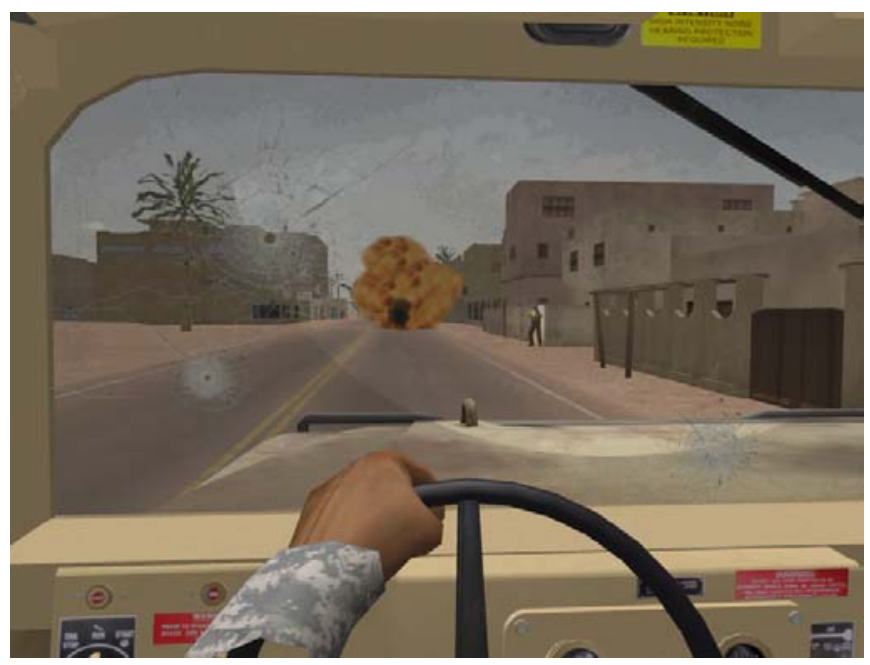

Fig. 6. IED Attack in Desert Road Humvee Scenario

usability of the prototype system application that fed an iterative design process. A clinical trial version of the application built from this process is currently being tested with PTSD-diagnosed personnel at a variety of sites. The primary test sites at the Naval Medical Center-San Diego (NMCSD) and at Camp Pendleton are conducting an open 
clinical trial to evaluate the system's efficacy for PTSD exposure therapy with active duty military personnel recently redeployed from Iraq.

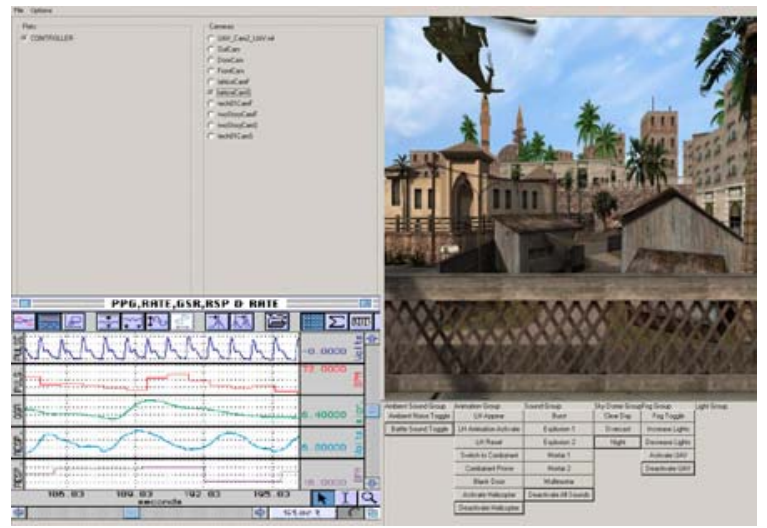

Fig. 7. "Wizard of Oz" Clinical Interface

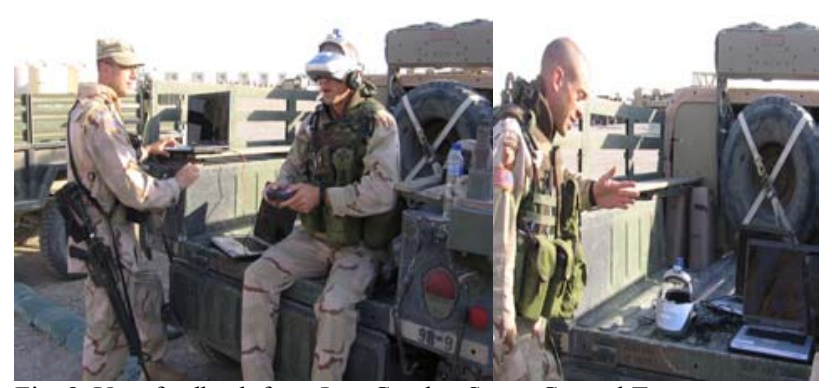

Fig. 8. User feedback from Iraq Combat Stress Control Team

The system is also being used to compare the efficacy of Cognitive Behavioral Therapy, VR and pharmacotherapy (Sertraline) alone and in combination, with placebo controls and blinded outcome measures at the Uniformed Services University of the Health Sciences. A randomized controlled trial comparing VR alone and VR+D-cycloserine is in progress at Emory University and a usability design trial is ongoing with returning soldiers at Ft. Lewis, Washington. Six other sites are also preparing to commence clinical treatment and testing with this system addressing a variety of research questions ranging from PTSD assessment to predicting future risk of developing PTSD using psychophysiological measures. The current paper will present two brief case reports from the first two treatment completers from the Naval Medical Center in San Diego. The presentation at the Virtual Rehabilitation 2007 conference will provide an updated set of results from all patients treated with the Virtual Iraq system at that time. As of the submission date for this paper (07/15/07), we have successfully treated five of seven active duty patients at NMCSD (See Figure 9) and one National Guard veteran was successfully treated as part of the Emory University study (See Figure 10) that has just begun.

\section{CASE STUDIES}

\section{A. Case 1}

The patient was a 22 year old, female Army private who met DSM-IV criteria for Posttraumatic Stress Disorder (PTSD) and Major Depressive Disorder, Recurrent (MDD). Her service in Iraq typically involved direct evaluation of locations immediately following suicide and/or IED bombings and she was exposed to significant human carnage during the course of her 1-year deployment. Upon returning stateside, following an evaluation, she was diagnosed with PTSD and agreed to participate in our standardized clinical research protocol. The protocol employs a ten-session treatment model that includes components of psychoeducation, initial imaginal exposure transitioning to prolonged virtual reality exposure therapy (VRET) and exposure-based homework exercises between sessions. Psychological assessment instruments administered were the PTSD Checklist Military Version (PCL-M) [16], the Patient Health Questionniare-9 (PHQ-9)[17] and the Beck Anxiety Inventory (BAI)[18]. Subjective Units of Distress (SUDs; 0100 scale) were gathered every five minutes during the virtual reality exposure and the homework included listening to the audiotapes of the patient's self-generated verbal narrative of her trauma relevant experiences while participating in the virtual reality exposure.

The patient showed a gradual and progressive improvement over the course of the VRET sessions. Scores on the PCL-M, PHQ-9, and BAI, prior to treatment were 42, 20 , and 12 , respectively. Post treatment scores on these measures decreased to 22, 3, and 0 . At follow-up, the subject did not meet DSM-IV diagnostic criteria for PTSD, and met remission status for MDD. The patient presented self-report and psychophysiological signs of habituation across VRET sessions and self-reported a concomitant decline across homework sessions while listening to the audiotape of her trauma narrative recorded during treatment sessions. For example, initial SUDs ratings while doing audiotape listening at home fell in the 30-35 range and declined to the 10-15 range at the end of treatment. Following completion of treatment, the patient was able to return to her unit and at three month follow-up, she continued to maintain the therapeutic gains observed at the end of treatment with scores on the PCL-M, PHQ-9 and BAI, at 18,1 , and 1 , respectively.

\section{B. Case 2}

The patient was a 29 year old, male U.S. Marine who was deployed to Iraq for seven months. Since returning to the USA, he suffered from PTSD for approximately six months. After a suicide attempt, the patient was psychiatrically hospitalized and diagnosed with Chronic PTSD. At that time, he was started on Sertraline and placed in a limited duty status that prevented him from returning to his unit of combat engineers.

The patient was initially contacted for participation in an open clinical trial using VRET. He participated in the initial 
assessment session, where he was administered the PCL-M, PHQ-9 and the BAI. Results from these tests confirmed the diagnosis of PTSD, and indicated that the patient had ongoing significant symptoms of PTSD, depression, and anxiety. However, the patient dropped out before treatment began, stating that he was unwilling to participate in the formal, structured study. However, he reported that he still wanted to participate in VRET, as dictated by clinical judgment rather than within the standard study protocol that required a commitment to 10 VRET sessions. After some negotiation, the patient participated one session of general, supportive therapy by phone lasting approximately one hour. Following this he agreed to participate further and was then seen for six, bi-weekly, 90-120 min sessions that incorporated supportive therapy, traditional imaginal exposure therapy, and VRET.

Despite the appearance of significant, new interpersonal stressors during treatment, the patient showed a rapid and dramatic improvement and no longer reported to be suicidal. Self-report and physiological (heart rate, GSR, respiration) responses in the Virtual Iraq simulation of combat indicated a progressive habituation effect across sessions and by the end of treatment, the patient showed little distress or abnormal physiologic reactivity despite maximal stimulation in the VR environment. Scores on the PCL-M, PHQ-9, and BAI, prior to treatment were 62,16 , and 28 , respectively. Post treatment scores on these measures decreased to 37,5 , and 22. Based on these indicators, he was tentatively judged to been adequately treated and was returned to his unit's psychiatrist for a fitness for duty evaluation. Independent evaluation determined that the patient was fit for full duty and he was returned for duty with his previous unit. One month after the completion of treatment, a repeat evaluation was performed which showed ongoing remission of PTSD symptoms and that the patient was functioning well in his previous military duties. Post-treatment scores on the PCLM, PHQ-9, and BAI were 21, 4, and 15, respectively, all in the sub-clinical range. A phone interview at three months post-treatment indicated that the patient was continuing to function well and this report was also further corroborated by family members.

\section{CONCLUSIONS}

The two clinical cases presented in this paper were the first two patients treated at the Naval Medical Center in San Diego and Camp Pendleton, and are initial positive indicators of the promise of this treatment. At the time of this writing, we have successfully treated five of seven active duty patients at NMCSD (See Figure 9) and one National Guard veteran was successfully treated as part of the Emory

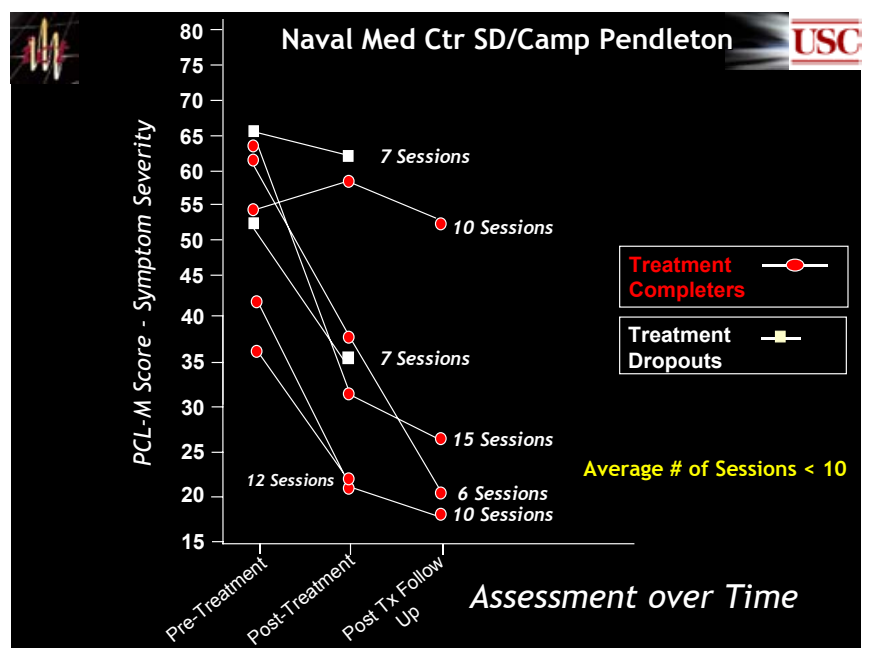

Fig. 9. Treatment results from NMCSD and Camp Pendleton as of 7/2007

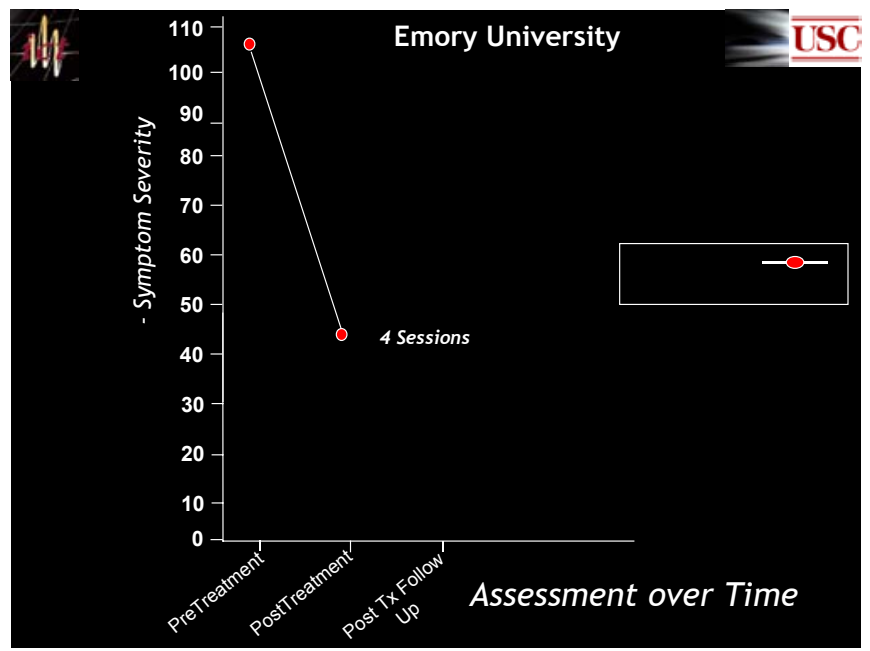

Fig. 10. Treatment result from first patient in Emory University Study

University study (See Figure 10). However, it should be noted that two other participants have recently chose to discontinue treatment before completion (both seven sessions each-one with clinical gains and one without) and another patient recently completed treatment with no observed therapeutic benefits. Results from such initial case reports are difficult to generalize from and we are cautious not to make excessive claims based on these early results. At the current time we are encouraged by these early successes and we continue to gather feedback from the patients regarding the therapy and the Virtual Iraq environment in order to continue our iterative system development process. We are in fact releasing an updated version of the system in June 2007 with added functionality that has its design "roots" from feedback acquired from these initial patients and the therapists who have used the system thus far. It should also be noted that this project is currently in an open clinical trial phase. As such, we intend to use such initial results to develop, explore and test hypotheses as to how we can improve treatment, and most importantly, try to determine what patient characteristics may predict who will benefit from VRET and who may be best served by other approaches. 
The current clinical treatment research program with the Virtual Iraq application will also provide important data that will determine the feasibility of expanding the range of research questions that can be addressed from this initial research and development program. An ongoing design principle in the iterative evolution of the Virtual Iraq system has always focused on the creation of a VR system/tool that could address both clinical and scientific research questions in the area of PTSD in a more comprehensive fashion. In this regard, we intend to expand our research program in the following areas:

- The use of the Virtual Iraq system to study the feasibility of assessing soldiers in advance of deployment to predict those that might have a higher likelihood of developing PTSD or other mental health difficulties based on physiological reactivity (and other measures) to a series of virtual combat engagements.

- The use of the system to deliver "stress inoculation" training to better prepare military personnel for what might occur in real combat environments.

- To study the effectiveness of using Virtual Iraq as an assessment tool that is administered immediately upon redeployment in order to determine who may be "at risk" for developing full-blown PTSD after an incubation period. Psychophysiological reactivity would figure well as a marker variable for this project and a prospective longitudinal study is needed in this area. This is particularly important for maximizing the probability that a soldier at risk would be directed into appropriate treatment or programming before being sent on a $2^{\text {nd }}$ or $3^{\text {rd }}$ deployment.

- To study the impact of multiple traumatic events on the course of PTSD as may be relevant for the reintegration of military personnel into civilian settings following multiple deployments.

- To study the differences between National Guard, reservist personnel and members of the regular standing military in terms of their susceptibility for developing PTSD and to examine how the course of treatment should be conducted. This rationale is also relevant for the study of PTSD differences due to age, gender, education, family support, and previous exposure to trauma (as in the case of a reservist who served in emergency services as a civilian in the police or fire dept. where exposure to traumatic events commonly occurs).

- To evolve understanding of the neuroscience of PTSD via the use of brain imaging protocols (fMRI, MRI, DTI, PET, fNIRS, etc.), traditional physiological measurement (EEG, EKG, GSR, etc.) and other forms of body-based responses (eyeblink and other motor behaviors) by leveraging the high controllability of stimulus events that is available with the Virtual Iraq application.
- To study the treatment efficacy of our VR system across a range of standard therapeutic issues (how much therapy, what rate of exposure is needed to optimally treat PTSD, value of a multi-component approach via dismantling studies).

- To study the interaction between the use of VR exposure in combination with a host of pharmacological treatment strategies.

- To expand the functionality of our existing system based on the results of the ongoing and future research. This will involve refining the system in terms of the breadth of scenarios, the audio and visual stimulus content and the level of Artificial Intelligence built into virtual human characters that function in the system.

One of the more foreboding findings in the Hoge et al., [1] report, was the observation that among Iraq/Afghanistan War veterans, “...those whose responses were positive for a mental disorder, only 23 to 40 percent sought mental health care. Those whose responses were positive for a mental disorder were twice as likely as those whose responses were negative to report concern about possible stigmatization and other barriers to seeking mental health care." (p. 13). While military training methodology has better prepared soldiers for combat in recent years, such hesitancy to seek treatment for difficulties that emerge upon return from combat, especially by those who may need it most, suggests an area of military mental healthcare that is in need of attention. To address this concern, a VR system for PTSD treatment could serve as a component within a reconceptualized approach on how treatment is accessed by veterans returning from combat. Perhaps VR exposure could be embedded within the context of "post-combat reintegration training" whereby the perceived stigma of seeking treatment could be lessened as the soldier would be simply involved in this "training" in similar fashion to other designated duties upon redeployment. VR PTSD therapy may also offer an additional attraction and promote treatment seeking by certain demographic groups in need of care. The current generation of young military personnel, having grown up with digital gaming technology, may actually be more attracted to and comfortable with participation in a VR application approach as an alternative to what is viewed as traditional "talk therapy" (even though such talk therapy would obviously occur in the course of a recommended multi-component approach for this disorder). It has been generally reported by practitioners who use VR to treat civilians with simple phobias, that patients who have avoided therapy for years, will sometimes choose to seek VR exposure treatment, perhaps due to a reduced perception of stigma. Reger et al., [19], has recently reported a similar attitudinal propensity in military personnel; among those who reported a disinclination to seek standard mental health treatment, 19\% rated a VR approach more favorably.

Finally, one of the guiding principles in our development work concerns how new Virtual Reality systems can extend the skills of a well-trained clinician. VR exposure therapy 
approaches are not intended to be automated treatment protocols that are administered in a "self-help" format. The presentation of such emotionally evocative VR combatrelated scenarios, while providing treatment options not possible until recently, will most likely produce therapeutic benefits when administered within the context of appropriate care via a thoughtful professional appreciation of the complexity and impact of this disorder.

\section{REFERENCES}

[1] C.W. Hoge, C.A. Castro, S.C. Messer, D. McGurk, D.I. Cotting, and R.L. Koffman, "Combat Duty in Iraq and Afghanistan, Mental Health Problems, and Barriers to Care," New England Jour of Medicine, vol. 351, no. 1, pp. 13-22, 2004.

[2] K.H. Seal, D. Bertenthal, C.R. Nuber, S. Sen, and C. Marmar, "Bringing the War Back Home: Mental Health Disorders Among 103,788 US Veterans Returning From Iraq and Afghanistan Seen at Department of Veterans Affairs Facilities," Arch Intern Med, vol. 167, pp. 476-482, 2007.

[3] C.W. Hoge, J.L. Auchterlonie, and C.S. Milliken, "Mental Health Problems, Use of Mental Health Services, and Attrition From Military Service After Returning From Deployment to Iraq or Afghanistan," JAMA, vol. 295, no. 9, pp. 1023-1032, 2006.

[4] DSM-IV, American Psychiatric Association, Washington, D.C., 1994

[5] R.A. Bryant, "Psychosocial Approaches of Acute Stress Reactions," CNS Spectrums, vol. 10, no. 2, pp. 116-122, 2005.

[6] B.O. Rothbaum, E.A. Meadows, P. Resick, and D.W. Foy, "Cognitive-Behavioral Treatment Position Paper Summary for the ISTSS Treatment Guidelines Committee," Journal of Traumatic Stress, vol. 13, pp. 558-563, 2000.

[7] B.O. Rothbaum, L. Hodges, D. Ready, K. Graap, and R. Alarcon, "Virtual reality exposure therapy for Vietnam veterans with posttraumatic stress disorder," Journal of Clinical Psychiatry, vol. 62, pp. $617-622,2001$

[8] B.O. Rothbaum, and A. Schwartz, "Exposure therapy for posttraumatic stress disorder," American Journal of Psychotherapy, vol. 56 , pp. 59-75, 2002

[9] J. Difede, and H. Hoffman, "Virtual reality exposure therapy for World Trade Center Post Traumatic Stress Disorder," Cyberpsychology and Behavior, vol. 5, no. 6, pp. 529-535, 2002.

[10] L.H. Jaycox, E.B. Foa, and A.R. Morral, "Influence of emotional engagement and habituation on exposure therapy for PTSD," Journal of Consulting and Clinical Psychology, vol. 66, pp. 186-192, 1998.

[11] K. Glantz, A.A. Rizzo, and K. Graap, "Virtual Reality for Psychotherapy: Current Reality and Future Possibilities," Psychotherapy: Theory, Research, Practice, Training, vol. 40, no. 1/2, pp. 55-67, 2003.

[12] A.A. Rizzo, M.T. Schultheis, K. Kerns, and C. Mateer, "Analysis of Assets for Virtual Reality Applications in Neuropsychology," Neuropsychological Rehabilitation, vol. 14 no. 1, pp. 207-239, 2004.

[13] J. Difede, J. Cukor, I. Patt, C. Goisan, and H. Hoffman, "The Application of Virtual Reality to the Treatment of PTSD Following the WTC Attack," Annals of the New York Academy of Sciences, vol. 1071, pp. 500-501, 2006.

[14] B. Rothbaum L. Hodges, R. Alarcon, D. Ready, F. Shahar, K. Graap, J. Pair, P. Hebert, D. Gotz, B. Wills, and D. Baltzell, "Virtual reality exposure therapy for PTSD Vietnam veterans: A case study," Journal of Traumatic Stress, vol. 12, pp. 263-271, 1999.

[15] A.A. Rizzo, B.O. Rothbaum, and K. Graap, "Virtual Reality Applications for Combat-Related Posttraumatic Stress Disorder," in Combat Stress Injury Theory, Research and Management, C.R. Figley and W.P. Nash, Eds. New York: Taylor and Francis Books, 2006, pp. 183-204.

[16] D. Forbes, M. Creamer, and D. Biddle, "The validity of the PTSD checklist as a measure of symptomatic change in combat-related PTSD," Behav Res Ther, vol. 39, no. 8, pp. 977-986, 2001.

[17] K. Kroenke, R.L. Spitzer, and J.B. Williams, "The PHQ-9: validity of a brief depression severity measure," J Gen Intern Med, vol. 16, no. 9, pp. 606-613, 2001.
[18] A. Osman, B.A. Kopper, F.X. Barrios, J.R. Osman, and T. Wade, "The Beck Anxiety Inventory: reexamination of factor structure and psychometric properties," J Clin Psych., vol. 53, no. 1, pp. 7-14, 1997.

[19] G.M. Reger, G.A. Gahm, A.A. Rizzo, R.A. Swanson, J. Etherage, and M.A. Reger, "Virtual reality in operational and garrison psychology: A review of the applications of the VR Iraq at Fort Lewis," Presented at the Cybertherapy 2007 Conference, Wash. D.C., June 11-14, 2007. 